\title{
La investigación cualitativa
}

\section{Qualitative research}

Ing. María Auxiliadora Guerrero Bejarano, MBA

Universidad Internacional del Ecuador, Ecuador

Autor para correspondencia: maguerrero@mail.internacional.edu.ec

Fecha de recepción: 15 de enero de 2016 - Fecha de aceptación: 15 de febrero de 2016

\section{Resumen}

La investigación cualitativa es uno de los tipos de investigación más usado. Este estudio analiza las bases de la investigación cualitativa, sus enfoques, planeamiento y herramientas necesarias para su buena implementación. El propósito del estudio es poder comprender y demostrar la fortaleza de esta metodología de investigación.

Palabras claves: investigación cualitativa; muestreo; herramientas de investigación

\begin{abstract}
Qualitative research is one of the most utilized research types. This study analyzes the fundamentals of qualitative research, its focuses, and the necessary planning and tools for its effective implementation. The purpose of the study is to understand and demonstrate the strength of this research methodology
\end{abstract}

Key words: qualitative research; sample; research tools 


\section{Introducción}

La Investigación Cualitativa o metodología Cualitativa es un método de investigación que se utiliza principalmente en las Ciencias Sociales. Se desarrolla a través de metodologías basadas en principios teóricos como la fenomenología que según la Filosofía Contemporánea es la práctica que aspira al conocimiento estricto de los fenómenos, que son simplemente las cosas tal y como se muestran y ofrecen a la consciencia. En este documento encontraremos el análisis del método cualitativo de investigación, sus procesos y paradigmas.

Los Investigadores Cualitativos tienen como objetivo principal hacer comprensible los hechos. Tradicionalmente se ha creído que la Investigación Cualitativa es una contraparte de la Investigación Cuantitativa, cuando en realidad las dos se complementan si el Investigador puede establecer desde un principio de su Investigación los objetivos de la misma.

Bonilla, E. \& Rodríguez, P. (2005) definen que el método cualitativo se orienta profundizar casos específicos y no a generalizar. Su preocupación no es prioritariamente medir, si no cualificar y describir el fenómeno social a partir de los rasgos determinantes, según sean percibidos por los elementos mismos que están dentro de la situación estudiada.

Bernal, C (2010) define que los investigadores que utilizan el método cualitativo buscan entender una situación social como un todo, teniendo en cuenta sus propiedades y su dinámica. En su forma general, la investigación cuantitativa parte de cuerpos teóricos aceptados por la comunidad científica, en tanto que la investigación cualitativa pretende conceptuar sobre la realidad, con base en la información obtenida de la población o las personas estudiadas.

Se puede concluir que la Investigación Cualitativa es utilizada generalmente en el análisis de las Ciencias Sociales, siendo un proceso metodológico que utiliza como herramientas a las palabras, textos, discursos, dibujo, gráfico e imágenes (datos cualitativos) para comprender la vida social por medio de significados, desde una visión holística, es decir que trata de comprender el conjunto de cualidades que se al relacionarse producen un fenómeno determinado.

Los sucesos sociales son muy diversos, sus modelos y las posibles combinaciones los hacen ilimitados en las posibles variaciones en las que se presentan. En ocasiones una teoría ya existente puede resultar deficiente para intentar explicar un fenómeno social, en esos casos se la debe construir desde un análisis cualitativo profundo, que pueda sustentar o rechazar las proposiciones propuestas por el Investigador. La Investigación Cualitativa, es concebida como una propuesta ontológica, epistemológica y se caracteriza porque genera un gran número de interpretaciones a través de los métodos que utiliza. Generalmente el método cualitativo asume la inducción como vía para el desarrollo de criterios que permitirán determinar la validez de los diferentes conocimientos.

\section{El enfoque cualitativo: sus comienzos}

Hernández, R., Fernández, C \& Batptista, P. (2010) ubican sus orígenes en distintos momentos: (a) los sitúan en los siglos XV y XVI con la denominada etnografía temprana, en la que se estudiaba a los pueblos primitivos; (b) fundamentalmente los circunscriben a principios 
del siglo XX; (c) se inicia como un proceso investigativo a finales del siglo XIX y sobre todo en el comienzo del siglo XX, aunque algunos arqueólogos realizaron estudios inductivos en la mitad del XIX.

Bernal, C (210) encuentra como el primer problema que hay diversas visiones que se han considerado como tipos de investigación cualitativa y las bases epistemológicas son variadas, pero concuerda en que el constructivismo es tal vez el paradigma que influyó más en el enfoque cualitativo. Las cosas en sí mismas existen, pero nosotros las apreciamos del modo como es capaz de percibirlas nuestra mente. El constructivismo como uno de los "padres" del enfoque cualitativo le otorga los énfasis principales que lo caracterizan:

- El reconocimiento de que el investigador necesita encuadrar en los estudios los puntos de

- vista de los participantes.

- La necesidad de inquirir cuestiones abiertas.

- Dado que el contexto cultural es fundamental, los datos deben recolectarse en los lugares

- donde las personas realizan sus actividades cotidianas.

- La investigación debe ser útil para mejorar la forma en que viven los individuos.

- Más que variables “exactas” lo que se estudia son conceptos, cuya esencia no

- $\quad$ solamente se captura a través de mediciones.

\section{Esencia de la investigación cualitativa}

La investigación Cualitativa se centra en comprender y profundizar los fenómenos, analizándolos desde el punto de vista de los participantes en su ambiente y en relación con los aspectos que los rodean. Normalmente es escogido cuando se busca comprender la perspectiva de individuos o grupos de personas a los que se investigará, acerca de los sucesos que los rodean, ahondar en sus experiencias, opiniones, conociendo de esta forma cómo subjetivamente perciben su realidad.

\section{Planteamiento de una investigación cualitativa}

A pesar de que el Método Cualitativo es Inductivo, debemos conocer profundamente el tema que vamos a tratar y sus posibles dificultades en la búsqueda de información que necesitamos obtener para el desarrollo de la investigación.

El planteamiento cualitativo suele incluir: los objetivos, las preguntas de investigación, la justificación y la viabilidad, una exploración de las deficiencias en el conocimiento del problemas y la definición inicial del ambiente o contexto. Plantea que los propósitos de una investigación cualitativa son:

1. Plantear cada objetivo en una oración o párrafo por separado

2. Enfocarse en explorar y comprender un solo fenómeno, concepto o idea.

3. Usar palabras que sugieran un trabajo exploratorio ("razones", "motivaciones", "búsqueda", "indagación", etcétera)

4. Usar verbos que comuniquen las acciones que se llevarán a cabo para comprender el fenómeno. Por ejemplo, los verbos "describir", "entender", "desarrollar", etcétera, permiten la apertura y flexibilidad que necesita una investigación cualitativa.

5. Usar un lenguaje neutral, no direccionado. Evitar palabras (principalmente adjetivos 
cualitativos) que puedan limitar el estudio o implicar un resultado específico.

6. Si el fenómeno o concepto no es muy conocido, proveer una descripción general de éste con la que se estará trabajando.

7. Mencionar a los participantes del estudio (ya sea uno o varios individuos, grupos de personas u organizaciones). En ocasiones pueden ser animales o colectividades de éstos, así como manifestaciones humanas (textos, edificaciones, artefactos, etcétera).

8. Identificar el ligar o ambiente inicial del estudio. (Roberto Hernández, R. y otros, 2010)

Los Planteamientos Cualitativos se caracterizan por ser abiertos, expansivos, aunque a medida que se avanza en la investigación van enfocándose en conceptos relevantes.

Normalmente no están direccionados desde el inicio. Se fundamentan en la experiencia e intuición del investigador. Son aplicables a un menor número de casos. Se orientan a aprender de experiencias y puntos de vista de las personas, valoran procesos y generan teorías que se apoyan en los puntos de vistas de aquellos que participan en el estudio.

Se debe considerar además que a pesar de ser más generales los planteamientos cualitativos deben situarnos en tiempo y lugar (Hernández y otros, 2010). Al plantear el problema hay que considerar que la investigación cualitativa es conducida principalmente en los ambientes naturales de los participantes; las variables no son controladas ni manipuladas, debido a que generalmente son conceptos generales como emociones y vivencias.

Los significados de estos conceptos son generados por los participantes en la investigación; los datos no sé reducen a números. Adicional a medida que se van obteniendo los distintos datos, por medio del método de recolección de información determinado, en ocasiones deberemos ir modificando su análisis o enfoque de acuerdo a las necesidades del estudio. Los planteamientos cualitativos tienen elementos de justificación al igual que los cuantitativos, dichos elementos son: conveniencia, relevancia social, implicaciones prácticas, valor teórico y utilidad metodológica. Pero su flexibilidad es mayor.

En un estudio cualitativo la hipótesis tiene un papel diferente puesto que no se pueden establecer desde el inicio de la investigación, se van generando en el proceso de investigación. Al describir el ambiente en el que se desarrollará la investigación cualitativa se debe ser lo más detallista posible, para que las personas que lean la investigación puedan comprender de mejor forma los fenómenos que se intentan explicar o comprobar. Se deben tomar distintos tipos de datos, desde la observación directa, interpretativas, temáticas, personales y de reacción de los participantes.

Estos datos deben ser registrados y detallados utilizando herramientas que faciliten su lectura posteriormente, como cuadros, esquemas, listados, mapas, diagramas, etcétera. En algunas ocasiones necesitaremos complementar información y podremos utilizar métodos como entrevistas, recolección de documentos, etcétera.

\section{Los campos de la investigación cualitativa}

La Investigación Cualitativa se puede desarrollar en múltiples campos, establecidos por el análisis del mundo social, sus relaciones, valores, actitudes y creencias. Se pueden considerar 
como campos de la realidad social los siguientes: El conocimiento de fenómenos complejos de la realidad a partir de lo cual se pretende construir conceptos muy abstractos, como los sentimientos, emociones y pensamientos.

Conocer la naturaleza de un grupo que forma un todo muy específico. Pueden ser grupos profesionales, raciales, étnicos, familiares, gremiales, institucionales. La definición de grupos puede estar dada por diferentes aspectos que los vuelvan homogéneos, como costumbres o afecciones. Se orienta también al análisis de fenómenos muy subjetivos que pueden ser poco comunicables, porque son realidades muy sensibles para las personas involucradas; por ejemplo casos de violaciones, madres solteras, aborto, etcétera. Los Fenómenos más típicamente humanos, como la libertad, la elección, la creatividad, el amor, el sentido de la muerte, el entusiasmo, etcétera.

En esta línea se puede considerar temas como la sexualidad, prácticas de amor, tabúes que significan fenómenos que analizan lo que los humanos definimos como bien y mal. Se encuentran también estudios cualitativos que involucran temas como la religión, realidades que pueden resultar vergonzosas para ciertas comunidades, fuentes de motivación de los distintos grupos y demás características de las distintas multitudes que guían o norman sus comportamientos, formas de relacionarse y toma de decisiones de acuerdo con los aspectos que enmarcan su desarrollo.

\section{Métodos utilizados en las investigaciones cualitativas}

Rodríguez Gómez, G., Flores Gil, J \& García Jiménez, Eduardo (1996) determinan que la investigación cualitativa utiliza los siguientes métodos: (a) fenomenología: busca conocer los significados que los individuos dan su experiencia y lo importante es aprender el proceso de interpretación por el que la gente define su mundo y actúa en consecuencia, (b)Etnografía: la entendemos como el método de investigación por el que se aprende el modo de vida de una unidad social concreta., (c) teoría fundamentada: trata de descubrir teorías, conceptos, hipótesis y proposiciones partiendo directamente de los datos, y no de supuestos a priori, de otras investigaciones o de marcos teóricos existentes, (d) etnometodología: intenta estudiar los fenómenos sociales incorporados a nuestros discursos y nuestras acciones a través del análisis de las actividades humanas, (e) investigación - acción: es una forma de búsqueda auto reflexiva para perfeccionar la lógica y la equidad de las propias prácticas sociales o educativas, comprensión de estas prácticas y las situaciones en las que se efectúan, (f) método biográfico: pretende mostrar el testimonio subjetivo de una persona en la que se recojan tanto los acontecimientos como las valoraciones que dicha persona hace de su propia existencia.

\section{Fases de la investigación cualitativa}

1. Definición del problema. - ninguna investigación puede empezar a desarrollarse sino conocemos el tema o problema a tratarse, de manera concreta, en el caso de las investigaciones cualitativas esta definición es provisional debido a que una de las tareas de la investigación es precisamente averiguar si la definición del problema es la correcta. En estos casos entonces definir significa orientar el problema. 
2. Diseño de Trabajo. - en esta etapa se desarrolla un plan de trabajo, que va a incluir detalles como cronogramas de actividades y presupuestos, tareas y un esquema teórico explicativo. El Diseño establecido también debe ser los suficientemente flexible como para adaptarse a los posibles cambios que se puedan presentar en el transcurso de la investigación.

3. Recogida de datos. - encontramos diferentes técnicas que se aplican en este tipo de investigación para la recogida de datos. El principio que rige esta fase es el de inspección, es decir que el investigador debe buscar la mayor proximidad a la situación, a buscar el foco descriptivo con el fin de analizar a cada persona con el fenómeno de estudio.

4. Análisis de datos. - después de recoger los datos con la técnica escogida, se analizarán los resultados obtenidos, en algunos casos se reorganizará la información antes de interpretarla. Esta etapa consiste en desentrañar las estructuras de significación y determinar su campo social y alcance.

5. Informa y validación de la información. - uno de los puntos más importantes y delicados de cualquier tipo de investigación es garantizar su validez. Por esta razón, se busca desarrollar interpretaciones conceptuales de los hechos que están a mano, teniendo cuidado de no proyectar resultados de posibles manipulaciones de la información obtenida. El trabajo cualitativo consiste en inscribir y especificar, es decir establecer el significado que determinados actos sociales tienen para sus actores y enunciar lo que este hallazgo muestra de su sociedad, y en general de toda la sociedad.

\section{Técnicas cualitativas de investigación}

1. La observación. - el termino observación es definido por la Real Academia de la Lengua como la acción de examinar atentamente algo que nos rodea. Abrir los ojos para contemplar, buscar, explorar, admirar, comparar, etcétera, en conjunción holística con el resto de los sentidos, es algo más que pasear la mirada diaria por nuestro entorno sin objetivo explícito alguno. Observar para conocer. La Observación es considerada la médula espinal del conocimiento científico y se convierte también en el eje que articula la metodología de la investigación cualitativa. Esta técnica nos permite obtener información sobre un fenómeno o acontecimiento tal y como se produce. Es la técnica más recomendada para las investigaciones cualitativas. La observación tiene como procedimiento un carácter selectivo y su finalidad es obtener información de algún tema en específico, lo que implica que se debe tener alguna idea de lo que se va a observar.

La Observación se puede planear con una finalidad exploratoria, con el fin de obtener información que luego se podrá corroborar con alguna otra técnica. Su contexto puede ser local, o más amplio, pero cualquiera que sea debe estar determinado en la descripción del proceso. Se debe considerar que la observación debe ser planificada en función al tiempo que va a durar, así como los límites dentro de los que se sitúan los elementos observados.

2. La observación participante. - la observación participante es una técnica con breve historia, ciertamente se origina de la observación pura, pero se presenta por situaciones específicas que modifican la relación clásica del observador y los observados. En función de la dificultad de acceso a la situación social de estudio, el investigador adoptará una 
postura diferente en lo que podríamos considerar los extremos entre la observación y la participación.

La cooperación entre estos elementos en cuatro tipos básicos: a) participante total, b) participante como observador, c) observador como participante y d) observador total. El último sostiene la distancia demandada anteriormente con objeto de no influir en los sujetos sometidos a observación. Entre las características básicas de la observación participante, encontramos que el objeto de investigación debe ser ajeno al investigador, la convivencia en el sistema sometido a estudio, supone el pilar fundamental de la aplicación del método. El sentido de los comportamientos, actitudes, etcétera, han de ser vistos desde la perspectiva de los miembros de la comunidad sometida a observación. El proceso de interacción ha de basarse en preguntas abiertas, flexibles y oportunas y debe haber una continua redefinición de lo que es problemático, basada en la observación previa, en la información que se va adquiriendo a través del trabajo de campo. Se trabaja en el contexto de un diseño abierto. El observador podrá hacer uso de uno o varios papeles en relación a los observados.

3. La entrevista. - técnica en la que una persona (entrevistador) solicita información a otra (entrevistado) sobre un problema determinado, presupone entonces la existencia al menos de dos personas y la posibilidad de interacción verbal. Encontramos diferentes tipos de entrevistas, la entrevista estructurada, por ejemplo, es aquella que se desarrolla dentro de un marco más rígido, donde el entrevistador utiliza un formato de preguntas que no son cambiadas o irrespetadas en su orden o sentido. Las entrevistas no estructuradas se caracterizan porque el entrevistador a pesar de tener un objetivo sobre la información que desea obtener, no se rige a una estructura formal, sino que puede sondear razones y motivos, desarrollando nuevas interrogantes en el transcurso de la entrevista de acuerdo a sus necesidades. En este tipo de entrevista se necesita una relación especial entre los participantes, en un inicio habrá desconfianza entre las partes, pero se pueden utilizar estrategias como generar más de una entrevista y ser fiel a los comentario o respuestas que este dé. Se puede decir que existe una verdadera relación de confianza entre el entrevistador y el entrevistado cuando este último aporta información personal.

4. La entrevista grupal. - método utilizado y relacionado generalmente con estudios de marketing y de tinte político, consiste en generar con un cuestionario cerrado entrevistar a una o varias personas que han tenido una experiencia previa con una situación específica, esta puede ser un anuncio publicitario o el uso de algún servicio, por ejemplo. En este tipo de entrevista, el entrevistador tiene un papel prominente por lo que debe estar preparado para guiar, dinamizar, animar a los participantes a dar su opinión o respuestas sobre las preguntas del guión establecido previamente.

5. El cuestionario. - técnica que se utiliza mayormente en investigaciones cuantitativas, pero que pueden ser de gran utilidad para investigaciones cualitativas, se recomienda que los cuestionarios no tengan más de treinta preguntas y son utilizados para obtener las opiniones de grupos numerosos que podrían colaborar invirtiendo tiempo mínimo. El cuestionario debe ser planificado y diseñado con meticulosidad de tal forma que se pueda obtener la información necesaria. Existen tres tipos de preguntas: cerradas, abiertas y de opción múltiple que permiten conseguir distintos tipos de respuestas.

6. El grupo de discusión. - es un grupo entre seis y diez personas, desconocidas entre sí y 
dispuestas a discutir un tema bajo la dirección o control de un moderador. Esta técnica no es suficiente sino se logra afianzar en ella el fenómeno del grupo. El segundo objetivo es que el tema de discusión esté centrado en un tema social objeto del estudio.

Hay distintas características en esta técnica, por ejemplo, las personas deben mantener una relación directa cara a cara, como mencionamos antes, deben ser desconocidas, las discusiones que se puedan dar deben al como fin buscar consenso. El grupo de discusión pretende construir y garantizar un escenario en el que los participantes puedan expresar sus propias opiniones con libertad y de la manera más espontánea.

\section{Definición de la Muestra}

"Muestra. - en el proceso cualitativo es un grupo de personas, eventos, sucesos, comunidades, etcétera, sobre el cual se habrán de recolectar los datos sin que necesariamente sea representativo del universo o población que se estudia” (Hernández, R. y otros 2010)

Existen tres factores que intervienen para determinar el número de casos que se deben estudiar para definir la muestra inicial: (a) Capacidad operativa de recolección y análisis, el número de casos que podemos manejar de manera realista y de acuerdo con los recursos que dispongamos., (b) El entendimiento del fenómeno, el número de casos que nos permitan responder a las preguntas de investigación, que más adelante se denominará "saturación de categorías"; (c) La naturaleza del fenómeno bajo análisis, si los casos son frecuentes y accesibles o no, si el recolectar información sobre éstos lleva relativamente poco o mucho tiempo. En algunos casos en los estudios cualitativos la muestra planteada al inicio de la investigación puede ser distinta a la muestra final, se pueden añadir o excluir dependiendo de las necesidades del estudio. (Hernández, R. 2010)

\section{Análisis de datos cualitativos}

Esta etapa se realiza una vez obtenida toda la información de acuerdo al método que se haya escogido, teniendo en cuenta los objetivos de la investigación que pueden haber variado en el transcurso de la ejecución del mismo. Es uno de los momentos más importantes del proceso e implica trabajar con los datos, ordenarlos, sintetizarlos, homogenizarlos en unidades manejables y descubrir qué aportan para la investigación.

El sentido de esta etapa consiste en reducir, categorizar, clarificar, sintetizar y comprara la información con el fin de contar con una visión lo más completa posible de la realidad objeto de estudio. Con este análisis se pretende poder representar la investigación de manera numérica y a través de gráficos. Debemos recordar que desde el comienzo los estudios cualitativos deben ser orientados de manera flexible. Esta etapa no es realizada al final del estudio, se realiza durante el desarrollo completo del mismo, en la medida que se va desarrollando la investigación, de esta manera el investigador puede ir adaptando sus objetivos en base a las nuevas perspectivas que se puedan presentar en la evolución del estudio.

Proceso de análisis de datos en la metodología cualitativa: en el desarrollo de la investigación encontramos tres momentos interdependientes, el análisis exploratorio es el 
primero, que implica el análisis del contexto con un marco conceptual previo. El segundo es la descripción que nos lleva al examen de todos los segmentos de cada categoría con el fin de establecer patrones en los datos para poder reducir los mismos. El último momento es la interpretación que es el más arriesgado, dado que interpretar supone integrar, relacionar y establecer conexiones entre las diferentes categorías, así como comparaciones. Se espera en este momento creatividad y compromiso con una opción determinada, pero se corre el riesgo de equivocarse.

\section{Conclusión}

Podemos concluir que la Investigación Cualitativa tiene ilimitadas posibilidades para poder analizar los diferentes sucesos que se puedan presentar de acuerdo a cada caso o tipo de estudio. Nos permite investigar aspectos sociales del comportamiento humano que no se pueden valorar de forma sencilla e intentar comprenderlos. De la misma manera encontramos que sus procesos y metodologías son muy variados también, yo me atrevería a decir que en los estudios cualitativos, jamás encontraremos uno exactamente igual a otro porque siempre podrá variar el proceso de acuerdo a las características del tema u objeto del mismo.

En definitiva, todos los procesos que se presentan en este tipo de investigaciones se van estableciendo de acuerdo a las circunstancias, y a pesar de que como en todo estudio debemos tener claro cuáles son los objetivos que deseamos alcanzar, en el método cualitativo existe una mayor libertad y flexibilidad de adaptación por los resultados que se puedan ir obteniendo. Adicionalmente para poder comprobar una teoría con este tipo de estudio se debe aplicar más de un método de recolección de datos que permita volver los resultados lo más objetivos posibles, que pueda permitirnos comprobar o rechazar una hipótesis luego de analizar diversos resultados para evitar caer en errores por subjetividad.

\section{Bibliografía}

Bernal, C (2010) Metodología de la Investigación, (3ª ed.) Bogotá, Colombia: Pearson Bonilla, E. \& Rodriguez, P. (2005). Más allá del dilema de los métodos. Bogotá, Colombia: Nomos

Hernández, R., Fernández, C \& Batptista, P. (2010) Metodología de la Investigación, (5a ed.).

México DF, México: Mc Graw Hill

Mejía, J. (2004). Sobre la investigación cualitativa: Nuevos conceptos y campos de desarrollo. Investigaciones Sociales, 8(13), 277-299.

Rodríguez Gómez, G., Flores Gil, J \& García Jiménez, Eduardo (1996) Metodología de la investigación Cualitativa. Granada, España: Aljibe 